\title{
Convolutional neural network-based segmentation can help in assessing the substantia nigra in neuromelanin MRI
}

\author{
Alice Le Berre ${ }^{1,2} \cdot$ Koji Kamagata $^{1}$ (1) $\cdot$ Yujiro Otsuka ${ }^{1,3} \cdot$ Christina Andica $^{1} \cdot$ Taku Hatano $^{4} \cdot$ Laetitia Saccenti $^{1,2}$. \\ Takashi Ogawa ${ }^{4}$. Haruka Takeshige-Amano ${ }^{4}$ - Akihiko Wada ${ }^{1}$ • Michimasa Suzuki ${ }^{1}$ - Akifumi Hagiwara ${ }^{1}$. \\ Ryusuke Irie ${ }^{1}$ - Masaaki Hori ${ }^{1}$. Genko Oyama ${ }^{4}$ - Yashushi Shimo ${ }^{4}$. Atsushi Umemura ${ }^{5}$. Nobutaka Hattori ${ }^{4}$. \\ Shigeki Aoki ${ }^{1}$
}

Received: 4 June 2019 / Accepted: 1 August 2019 / Published online: 10 August 2019

(C) The Author(s) 2019

\begin{abstract}
Purpose This study aimed to evaluate the accuracy and diagnostic test performance of the U-net-based segmentation method in neuromelanin magnetic resonance imaging (NM-MRI) compared to the established manual segmentation method for Parkinson's disease (PD) diagnosis.

Methods NM-MRI datasets from two different 3T-scanners were used: a "principal dataset" with 122 participants and an "external validation dataset" with 24 participants, including 62 and 12 PD patients, respectively. Two radiologists performed SNpc manual segmentation. Inter-reader precision was determined using Dice coefficients. The U-net was trained with manual segmentation as ground truth and Dice coefficients used to measure accuracy. Training and validation steps were performed on the principal dataset using a 4-fold cross-validation method. We tested the U-net on the external validation dataset. SNpc hyperintense areas were estimated from U-net and manual segmentation masks, replicating a previously validated thresholding method, and their diagnostic test performances for PD determined.

Results For SNpc segmentation, U-net accuracy was comparable to inter-reader precision in the principal dataset (Dice coefficient: U-net, $0.83 \pm 0.04$; inter-reader, $0.83 \pm 0.04$ ), but lower in external validation dataset (Dice coefficient: U-net, $079 \pm 0.04$; inter-reader, $0.85 \pm 0.03$ ). Diagnostic test performances for PD were comparable between U-net and manual segmentation methods in both principal (area under the receiver operating characteristic curve: U-net, 0.950; manual, 0.948) and external (U-net, 0.944; manual, 0.931) datasets.

Conclusion U-net segmentation provided relatively high accuracy in the evaluation of the SNpc in NM-MRI and yielded diagnostic performance comparable to that of the established manual method.
\end{abstract}

Keywords Parkinson disease $\cdot$ Magnetic resonance imaging $\cdot$ Neural networks (computer) $\cdot$ Artificial intelligence

Electronic supplementary material The online version of this article (https://doi.org/10.1007/s00234-019-02279-w) contains supplementary material, which is available to authorized users.

Koji Kamagata

kkamagat@juntendo.ac.jp

1 Department of Radiology, Juntendo University Graduate School of Medicine, 2-1-1, Hongo, Bunkyo-ku, Tokyo 113-8421, Japan

2 Department of Radiology, Université Paris Descartes, 12 rue de l'Ecole de Medecine, 75006 Paris, France

3 Milliman Inc., Tokyo, Japan

4 Department of Neurology, Juntendo University School of Medicine, Tokyo, Japan

5 Department of Neurosurgery, Juntendo University School of Medicine, Tokyo, Japan

\section{Introduction}

Parkinson's disease (PD) is the second most common progressive neurodegenerative disease and affects 8.5 million individuals worldwide as of 2017 [1]. It is characterized by a progressive loss of dopaminergic neurons within the substantia nigra pars compacta (SNpc), considered to cause PD's classical motor symptoms [2]. Currently, PD diagnosis relies on the clinical features acquired from patient history and neurological examination; accurate diagnosis is difficult in early stages, with a misdiagnosis rate of approximately $25 \%$ [3]. Although $60-80 \%$ of the dopaminergic neurons of the SNpc are lost before any clinical symptoms appear [4], to date, conventional MRI has been unsuccessful in detecting 
pathological changes in the SNpc, compromising the effectiveness of prophylactic approaches and new therapies [5] which attempt to slow the neuronal loss. Therefore, objective PD biomarkers are urgently desired.

In routine clinical practice, the role of MRI in patients with Parkinson-like motor symptoms is today limited to ruling out atypical parkinsonisms [6]. Recently, among other promising approaches $[7,8]$ developed to detect neurodegeneration in the SNpc, neuromelanin-MRI (NM-MRI) was proposed to visualize neuromelanin, as its depigmentation is a key pathological feature of PD [9]. Iron-neuromelanin complexes stored inside healthy dopaminergic neurons have highly paramagnetic properties that increase the NM-MRI signal intensity through a combination of magnetization transfer and $\mathrm{T} 1 \mathrm{ef}-$ fects [10]. After neuronal death, unbound neuromelanin and iron become extracellular [11], contributing to neurodegeneration by activating the microglia and proinflammatory factors [12]. In patients with PD, low levels of intracellular ironneuromelanin complexes result in decreased NM-MRI signal intensity. Several authors showed that quantifying the SNpc signal loss in NM-MRI can yield high diagnostic accuracy for distinguishing PD patients from controls [13-15], even at an early stage [16]. Furthermore, some studies reported a correlation with the severity of the disease $[17,18]$ and L-dopa induced motor complications [19].

To that purpose, various segmentation techniques have been proposed for assessing the hyperintense area of the SNpc: simple manual delineation [14], SNpc hyperintense area (or volume) estimation using a signal intensitythreshold derived from the manually segmented background midbrain $[15,18,19]$, and the semiautomated region growing technique [20]. The only automated process described to date is the atlas-based method [13], which involves aligning new images to a set of manually labeled examples. However, this method may not be able to capture the full anatomical variability of the target subjects due to the use of a fixed set of atlases, affecting its accuracy [21], and is known to be computationally intensive.

In this study, we used as reference a threshold signal intensity method using manual segmentation (MS) first described by Schwarz et al. [15], as it is the only method demonstrating a stage-dependant SNpc signal loss in PD, unlike the atlasbased experiment. This method attempts to count the SNpc hyperintense pixels above a determined threshold based on the background signal of the midbrain. Several steps, including manually delineating the SNpc and midbrain, determining the threshold, and calculating the resulting hyperintense areas, are required. Despite attractive diagnostic performances, the clinical applicability of this method is impeded by these timeconsuming steps, first and foremost MS; in this regard, automatized segmentation would be a significant improvement.

In this context, deep learning segmentation appears as an appealing option. It uses neural networks trained to perform tasks using examples and to grasp intricate structures in datasets [22]. Specifically, convolutional neural networks (CNNs) have significantly advanced computerized image recognition performance. They have successfully been applied to the neuroradiology field to segment various structures such as brain tumors [23], white matter hyperintensities [24], or organs-at-risks prior to radiation therapy [25]. Among CNNs, the U-net [26] is the most commonly used model in biomedical image segmentation.

We hypothesized that a U-net architecture CNN could replace manual segmentation of NM-MR images as the initial step of a previously described method aiming to assess $\mathrm{SNpc}$ signal intensity and achieve equivalent diagnostic accuracy for PD diagnosis. Therefore, we evaluated (1) the segmentation accuracy and (2) the diagnostic test performance of the Unet segmentation-based method compared to the established MS method.

\section{Methods}

\section{Study design and participants}

This retrospective case-control study used two NM-MRI datasets. A principal dataset from 60 patients with PD and 62 age- and gender-matched healthy controls (HC) was obtained by a 3T scanner (MAGNETOM Prisma, Siemens Healthcare) from October 2017 to July 2018 and was used to train and validate the U-net model. An external validation dataset, including 12 patients with PD and $12 \mathrm{HC}$, was obtained using a different 3T scanner (Achieva, Philips Medical Systems) from April 2014 to April 2015 and used to test the U-net. All patients were from the Neurology Department of Juntendo University Hospital and satisfied the Movement Disorder Society diagnostic criteria for clinically established $\mathrm{PD}$ [27]. These patients responded to antiparkinsonian therapy and remained free of atypical parkinsonism for 18 months or longer after being scanned. The HC group had no history of neurologic or psychiatric disorders. All the participants provided informed consent before examination and the Ethics Committee of the Juntendo University School of Medicine approved the study.

Using two 3T MR scanners, we obtained modified NMsensitive T1-weighted fast-spin echo sequences with additional spectral presaturation inversion-recovery pulses, similar to that proposed by Schwarz et al. [15]. General scan parameters for the principal data set were as follows: 600/12 ms repetition time/echo time; echo train length of $14 ; 2.5 \mathrm{~mm}$ slice thickness; $0.5 \mathrm{~mm}$ slice gap; $3.0 \mathrm{~mm}$ spacing between slices; $512 \times$ 359 acquisition matrix; $220 \times 220 \mathrm{~mm}$ field of view $(0.43 \times$ $0.43 \mathrm{~mm}$ pixel size); $175 \mathrm{~Hz} /$ pixel bandwidth, three-averages; 7:15 min of total scan time, whereas those for the external validation dataset were as follows: $688 / 15 \mathrm{~ms}$ repetition 
time/echo time; echo train length of 14; $3 \mathrm{~mm}$ slice thickness; $1.0 \mathrm{~mm}$ slice gap; $4.0 \mathrm{~mm}$ spacing between slices; $0.43 \times$ $0.43 \mathrm{~mm}$ pixel size; four-averages; 7:46 min of total scan time. In both cases, all the oblique-axial slices ranged from the splenium of the corpus callosum to the inferior border of the pons and were parallel to the line connecting the splenium to the genu of the corpus callosum and perpendicular to the fourth ventricle floor.

\section{Thresholding method based on manual segmentation}

We used a similar, slightly modified version of the method reported by Schwarz et al. [15] to measure the hyperintense area in the SNpc. Image analyses were performed by two radiologists blinded to clinical information [reader 1 (KK) and reader $2(\mathrm{AL})$ ] on an offline Windows computer using the MRIcron software v2010. In this study, we used the masks delineated by the most experienced radiologist (reader 1) as criterion standard for SNpc and midbrain segmentation.

First, masks were generated by manually delineating the SNpc and midbrain in two consecutive axial slices that included the midbrain (Fig. 1). Then, we measured the average background signal and standard derivation (SD) for each patient, where the background was defined as the midbrain subtracted by the SNpc. The hyperintense areas of the SNpc were calculated by multiplying the number of pixels within the SNpc masks exhibiting signals above a chosen threshold by the image resolution. The optimal threshold was determined in the principal dataset by performing ROC analyses for the SNpc hyperintense areas using several thresholding values with intervals of the same order of magnitude as previous authors [18, 19]:
$\mathrm{MSI}+1,1.5$, or $2 \mathrm{SD}$. The highest diagnostic accuracy was yielded using the MSI +1.5 SD threshold. We employed the same $+1.5 \mathrm{SD}$ threshold in the external validation dataset, on the principle of externalizing both the segmentation and thresholding processes as a whole. This manual process took approximately 5 to $10 \mathrm{~min}$ for each subject.

\section{U-net architecture CNN-based segmentation}

Deep learning segmentation was performed using a U-net architecture CNN [26] according to the maximum probability of candidate classes of each voxel. First, we augmented the principal dataset by changing the signal intensity, rotation degree, and scale of the original images to obtain a model that is robust against deviations, without prior signal intensity normalization. For signal intensity deviation, we converted the original signal intensity as

$A($ orig $+B)$,

where $A=0.7 / 1.0 / 1.3, B=-100 / 0 / 100$, and orig denotes the signal intensity of the original image.

For rotational deviation, we processed the original image by rotating it by $-30^{\circ} / 0^{\circ} / 30^{\circ}$. Finally, for scale deviation, we resized the original images to $90 \% / 100 \% / 110 \%$. We used these augmented datasets as the final training data. Two Unets were employed to perform two successive segmentation tasks, where one task was trained to segment the midbrain and the other was trained to segment the SNpc and background from the output of the former (i.e., midbrain $=\mathrm{SNpc}+$ background). The two U-nets had the same architecture, which differed slightly from the original U-net architecture [26].

\section{Principal Dataset}

HC

PD
DL
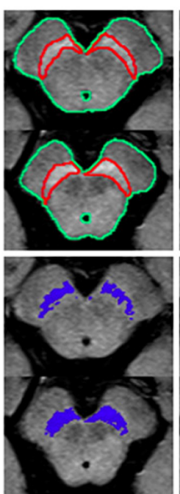

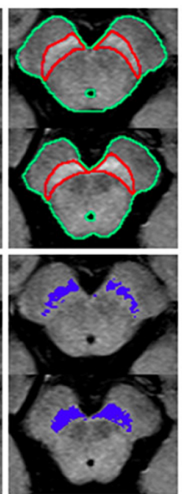

Manual

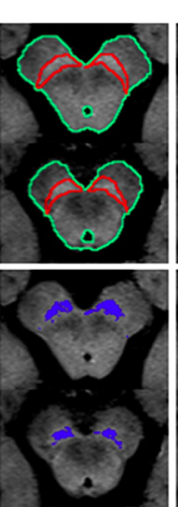

Fig. 1 Manually labeled neuromelanin-sensitive MR images of midbrain and masks of hyperintense area within the SNpc obtained using the mean

\section{External Validation Dataset}

$\mathrm{HC}$

$\mathrm{DL}$

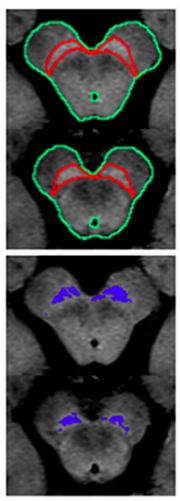

Manual
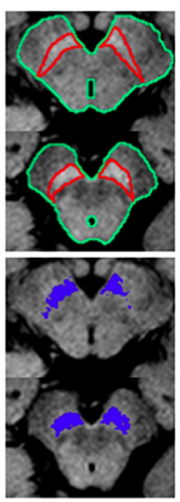

DL

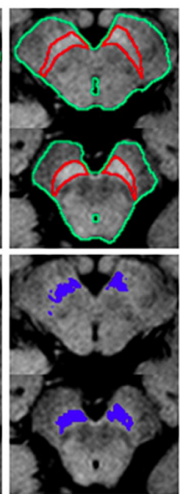

Manual

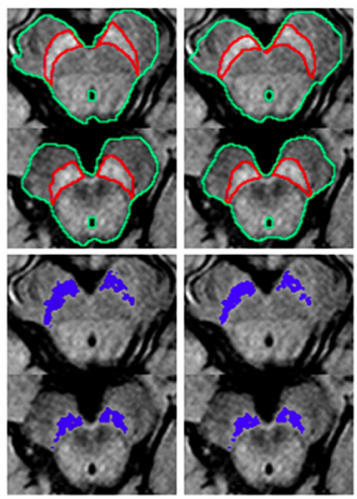

background signal intensity $+1.5 \mathrm{SD}$ as a threshold. $\mathrm{BG}$, background; DL, deep learning; HC, healthy control; PD, Parkinson's disease 
Here, max pooling operations were replaced by convolution with a stride of 2 . Both the U-nets were trained simultaneously to improve the second U-net's robustness to noise, as output images from the first U-net exhibited significant noise; however, the loss functions of these U-nets were disconnected, ensuring that the backpropagation of the loss function of one U-net did not affect the other. In one iteration, a $256 \times 256$ pixel image was randomly cropped from the original data $(512 \times 512$ pixels $)$ with a batch size of 2 , and mean cross entropy was computed for the loss function. The Adam [28] gradient descent algorithm was used to optimize the model. Here, the Adam update rule was applied with $\alpha=0.0001$, $\beta 1=0.5$, and $\beta 2=0.999$. Adam optimization was performed for 10 epochs. Training of the U-net was performed according to a 4-fold cross-validation based on four balanced groups mixing $\mathrm{HC}$ and PD patients with a 1:1 ratio. Validation and training Dice coefficients were similar in all folds; therefore, the model trained on the first fold was arbitrarily chosen to perform U-net segmentation (US). We did not employ early stopping or hyper parameter search.

As an inference phase, the trained U-net was applied to the external validation dataset to segment the unlabelled Achieva MR images. NM-rich areas of the SNpc were calculated using the optimal threshold obtained with the principal dataset (signal intensity of the midbrain +1.5SD).

A computer with 64 GB of CPU memory, a Xeon E5-2670 v3 CPU (Intel, Santa Clara, CA), and a TITAN Xp graphics processing unit (NVIDIA, Santa Clara, CA) was used to perform the model training. Python 3.6 and the DL framework of Chainer 3.2.0 (http://chainer.org/) was used to code the computer program. Each fold took $150 \mathrm{~min}$ to process. The time to predict (U-net segmentation and hyperintense voxels count) for each patient was less than $0.5 \mathrm{~s}$.

\section{Statistical analyses}

Statistical analyses were performed using the XLSTAT v2018.7 software. Age and gender distributions were compared between patients with PD and HC using Student's $t$ test and chi-squared test. The relative variation of the background signal was calculated by dividing the mean background signal with the standard deviation. The mean relative variations in the HC and PD groups were compared using the Student's $t$ test to assess the image quality. To evaluate the segmentation accuracy of the U-net, we used the Dice similarity coefficient (DSC) defined as Dice (MS, US) $=\frac{2|\mathrm{MS} \cap \mathrm{US}|}{|\mathrm{MS}|+|\mathrm{US}|}$, where MS denotes manual segmentation and US denotes U-net segmentation. DSC values range from 0 to 1 , where 0 and 1 indicate no and perfect overlapping, respectively. We compared the segmentation outputs of the U-net to the masks delineated manually by reader $1(\mathrm{KK})$, considered the criterion standard. We also compared the two readers' segmentation masks. The inter-reader precision and segmentation accuracy of the Unet was rated as follows: $0.0-0.39$, "low"; 0.40-0.79, "moderate"; and 0.80-1.0, "high." In terms of DSC, using MannWhitney $U$ test and Student's $t$ tests with the principal and external validation datasets, respectively, we compared the U-net accuracy or the inter-reader precision for SNpc or midbrain segmentation between the HC and PD groups. In addition, we compared the U-net accuracy to inter-reader precision for SNpc segmentation in both the HC and PD groups. The Student's $t$ test was used to compare the NM-rich areas of the SNpc between the PD and HC groups. The relations between the hyperintense areas and disease duration or UPDRS-III scores were determined using the Spearman's rank correlation test. The strength of the correlation was determined using the following criteria for correlation coefficient $\mathrm{r}$ : $0.00-0.19$, "very weak;" 0.20-0.39, "weak;" 0.40-0.59, "moderate;" $0.60-0.79$, "strong," $0.80-1.0$, "very strong." Finally, to evaluate the diagnostic performance of the thresholding method as a diagnostic test for PD using either manual or U-net segmentation, receiver operating characteristic (ROC) analyses of hyperintense SNpc areas were performed and areas under the curve (AUC) calculated. ROC curves form the same datasets were compared using the Delong method [29].

\section{Results}

\section{Participants and image quality}

There was no significant difference in age or gender between the $\mathrm{HC}$ and patients with PD in the two datasets (Table 1). Further, there was no significant difference in the relative variations of the background signals between the patients with $\mathrm{PD}$ and $\mathrm{HC}$ in both datasets for either segmentation method (all $p>0.05$, see supplemental data online), indicating that the image qualities were similar in the PD and $\mathrm{HC}$ groups.

\section{Evaluation of the U-net}

\section{Principal dataset}

Table 2 shows the inter-reader precision and US accuracy in terms of the DSC obtained for the SNpc and midbrain. The US accuracy of SNpc and midbrain (MB) was as high as the interreader precision, with similar DSCs for each subgroup and structure. The DSCs were consistently lower for the SNpc than for the midbrain (DSCs for all subjects: midbrain, 0.97 \pm 0.01 ; SNpc, $0.83 \pm 0.04)$. Further, regarding the SNpc, the DSCs were lower for patients with PD than HC. The calculated hyperintense areas within the SNpc were significantly lower in the patients with PD than in the HC [MS: PD, $48.6 \pm$ $19.1 \mathrm{~mm}^{3}$ (mean $\pm \mathrm{SD}$ ); HC, $84.9 \pm 14.4 \mathrm{~mm}^{3}$; US: PD, $45 \pm$ $18.5 \mathrm{~mm}^{3}$; HC, $83.9 \pm 14.5 \mathrm{~mm}^{3}$; all $p<0.0001$ using 
Table 1 Clinical characteristics of healthy controls and PD patients in the principal and external validation datasets

\begin{tabular}{llllllll}
\hline & \multicolumn{2}{l}{ Principal dataset } & & \multicolumn{3}{l}{ External validation dataset } \\
\cline { 2 - 3 } Variable & HC, $n=60$ & PD, $n=62$ & $p$ value & & HC, $n=12$ & PD, $n=12$ & $p$ value \\
\hline Gender $(\mathrm{m} / \mathrm{f})$ & $35 ; 25$ & $28 ; 34$ & 0.146 & & $12 ; 0$ & $12 ; 0$ & N.A. \\
Age, year & $70.82 \pm 3.68$ & $70.24 \pm 6.45$ & 0.549 & & $62 \pm 14.33$ & $62.5 \pm 9.69$ & 0.947 \\
Disease duration, year & N.A. & $9.98 \pm 6.22$ & & & N.A. & $15.75 \pm 13.33$ & \\
UPDRS-III score & N.A. & $22.47 \pm 15.67$ & & & N.A. & $20.08 \pm 9.88$ & \\
Hoehn and Yahr stage & N.A. & $2.73 \pm 0.87$ & & & N.A. & $2.5 \pm 0.52$ & \\
\hline
\end{tabular}

Data are presented as mean \pm standard deviation unless otherwise noted

$H C$, healthy controls; $P D$, Parkinson's disease; UPDRS-III, part III of the Unified Parkinson's Disease Rating Scale
Student's $t$ test] (Fig. 2a). Analysis of the correlation between the hyperintense area and PD duration demonstrated a significantly weak negative correlation with both methods (MS: $r=$ $-0.32, p=0.013$; US: $r=-0.33, p=0.008)$ but no significant correlation with the UPDRS-III score (MS: $r=-0.22, p=$ 0.079 ; US: $r=-0.20, p=0.110$ ). Using US instead of MS for the threshold signal intensity method did not affect the diagnostic test performance of NM-MRI. The AUCs for the hyperintense SNpc area were comparable using either the US or MS methods with a slight comparative advantage for US (AUCs, 0.950 and 0.948 , respectively, $p<0.05$, with optimal cut-off values of 61.2 and $64.2 \mathrm{~mm}^{2}$, Fig. 3).

\section{External validation dataset}

Visual assessment of the output segmentation maps did not reveal any large segmentation errors. The US accuracy for $\mathrm{SNpc}$ was high for $\mathrm{HC}$ and moderate for patients with $\mathrm{PD}$ but was significantly lesser than the inter-reader precision in both cases (mean DSCs: patients with PD, 0.77 versus 0.83 ; $\mathrm{HC}, 0.80$ versus 0.86 , all $p<0.0001$ ). As with the principal dataset, the SNpc segmentation was less accurate than the midbrain segmentation in each case (Table 2). Hyperintense areas were significantly lower in the patients with PD than
$\mathrm{HC}$, and the differences between these groups were similar with both methods [MS: PD, $43.9 \pm 18.2 \mathrm{~mm}^{3}$; HC, $87.6 \pm$ $23.1 \mathrm{~mm}^{3}$; US: PD, $36.1 \pm 17.4 \mathrm{~mm}^{3}$; HC, $80 \pm 23.7 \mathrm{~mm}^{3}$; all $p<0.0001$ using Student's $t$ test] (Fig. 2b). No significant correlation was found between the hyperintense area and disease duration (MS: $r=-0.24, p=0.449$; US: $r=-0.20, p=$ 0.527 ) with either method. However, there was a strong significant correlation with the UPDRS-III score (MS: $r=-0.65$, $p=0.027$; US: $r=-0.60, p=0.043)$. Here too, replacing MS by US did not seem to affect the overall diagnostic test performance of NM-MRI. The AUCs for the hyperintense SNpc area were respectively 0.944 and 0.931 when US or MS were employed $(p<0.05)$, with optimal cut-off values of 54.7 and $64.3 \mathrm{~mm}^{2}$ (Fig. 3).

\section{Discussion}

Here, we developed a U-net model to segment the SNpc and midbrain in NM-MRI and showed that our model could achieve equivalent diagnostic performance to that of manual segmentation using a validated thresholding method for the hyperintense area of the SNpc, despite a moderate segmentation accuracy of the SNpc by our model.

Table 2 Inter-reader precision and U-net segmentation accuracy shown as mean DSC obtained for the SNpc and midbrain in healthy controls and PD patients of both datasets

\begin{tabular}{|c|c|c|c|c|c|c|c|c|c|}
\hline \multirow[t]{2}{*}{ Dataset } & \multirow[t]{2}{*}{ Structure } & \multicolumn{4}{|c|}{$\begin{array}{l}\text { Mean DSC } \\
\text { Reader } 1 \text { versus reader } 2\end{array}$} & \multicolumn{4}{|c|}{$\begin{array}{l}\text { Mean DSC } \\
\text { U-net versus reader } 1\end{array}$} \\
\hline & & All & $\mathrm{HC}$ & PD & $p$ value* & All & $\mathrm{HC}$ & PD & $p$ value* \\
\hline Principal & $\begin{array}{l}\text { MB } \\
\text { SNpc }\end{array}$ & $\begin{aligned} 0.97 & \pm 0.01 \\
0.83 & \pm 0.04\end{aligned}$ & $\begin{array}{l}0.97 \pm 0.01 \\
0.85 \pm 0.04\end{array}$ & $\begin{array}{l}0.97 \pm 0.01 \\
0.81 \pm 0.04\end{array}$ & $\begin{array}{l}0.974 \\
<0.001\end{array}$ & $\begin{array}{l}0.97 \pm 0.01 \\
0.83 \pm 0.04\end{array}$ & $\begin{array}{l}0.97 \pm 0.01 \\
0.84 \pm 0.03\end{array}$ & $\begin{array}{l}0.97 \pm 0.02 \\
0.82 \pm 0.04\end{array}$ & $\begin{array}{l}0.868 \\
0.001\end{array}$ \\
\hline External validation & $\begin{array}{l}\text { MB } \\
\text { SNpc }\end{array}$ & $\begin{array}{l}0.96 \pm 0.02 \\
0.85 \pm 0.03\end{array}$ & $\begin{array}{l}0.95 \pm 0.02 \\
0.86 \pm 0.02\end{array}$ & $\begin{array}{l}0.96 \pm 0.02 \\
0.83 \pm 0.03\end{array}$ & $\begin{array}{l}0.563 \\
0.036\end{array}$ & $\begin{array}{l}0.95 \pm 0.01 \\
0.79 \pm 0.04\end{array}$ & $\begin{array}{l}0.96 \pm 0.01 \\
0.80 \pm 0.05\end{array}$ & $\begin{array}{l}0.95 \pm 0.02 \\
0.77 \pm 0.03\end{array}$ & $\begin{array}{l}0.532 \\
0.101\end{array}$ \\
\hline
\end{tabular}

Data are presented as mean \pm standard deviation unless otherwise noted

$D S C$, Dice similarity coefficient; $H C$, healthy controls; $M B$, midbrain; $P D$, Parkinson's disease; $S N p c$, substantia nigra pars compacta

*Between $\mathrm{HC}$ and PD patients 


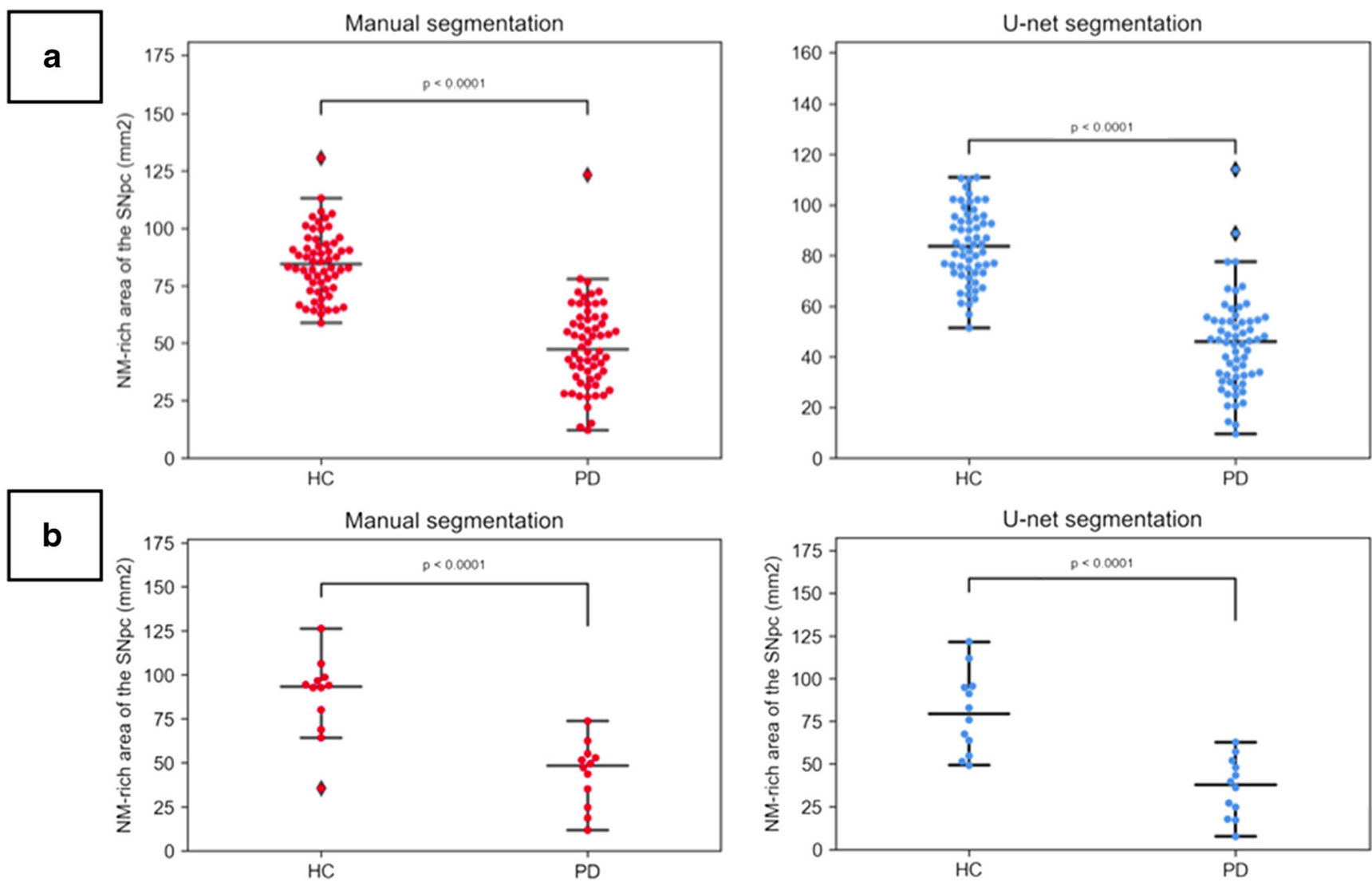

Fig. 2 Neuromelanin-rich areas obtained with manual (reader 1) and U-net segmentation methods on neuromelanin-sensitive MR images of the principal (a) and external validation (b) datasets

U-net segmentation of the midbrain was highly accurate in both datasets; however, the U-net could not achieve a segmentation of the SNpc in the same range as the inter-reader precision in the external validation dataset. The lower accuracy of the US for SNpc in the external dataset implies that different imaging parameters and signal intensity variations
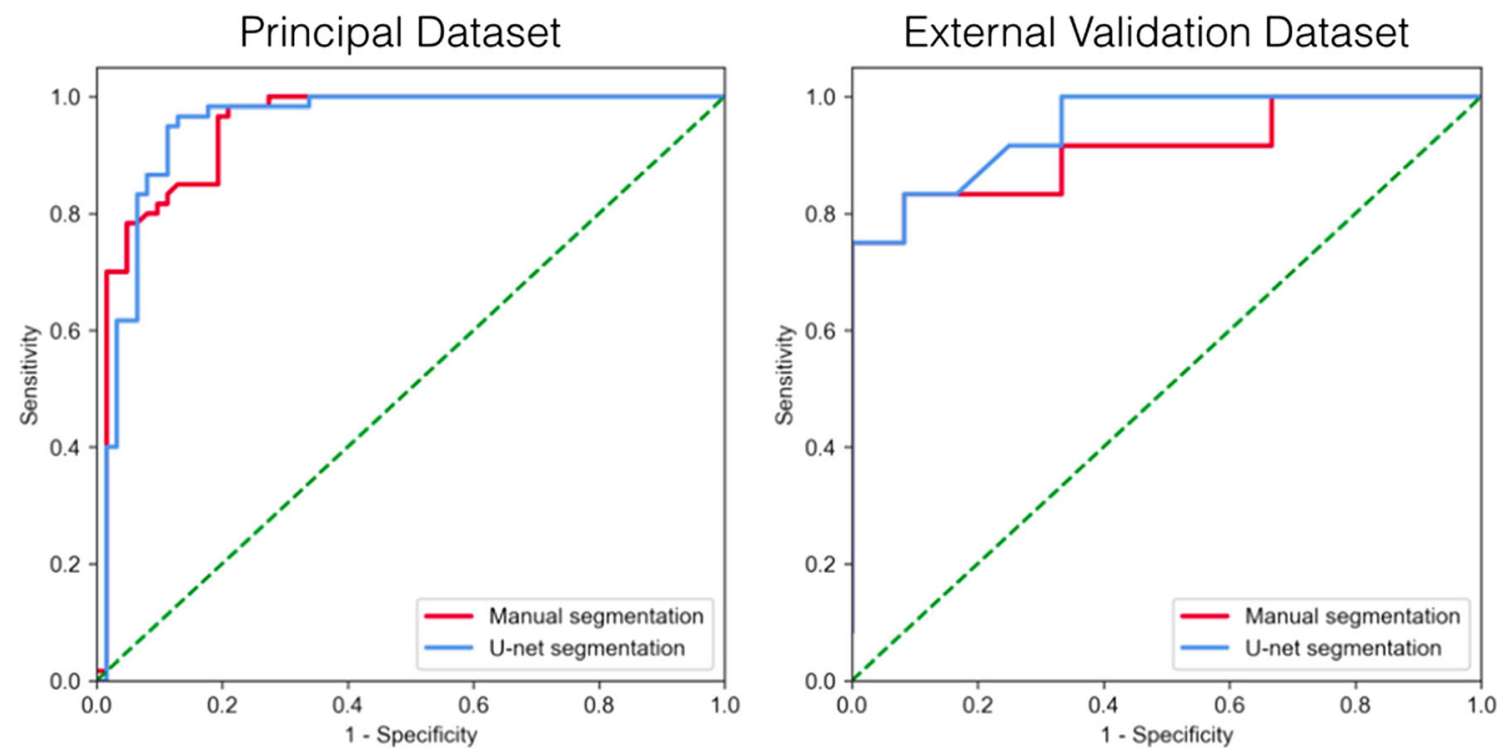

Fig. 3 ROC curves of NM-rich areas of the SNpc obtained by manual and U-net segmentations for differentiating patients with PD and healthy subjects in both datasets 
challenge the U-net inference capabilities. Also, applying the optimal threshold for the principal dataset to the external dataset could have affected the diagnostic test accuracy, because the threshold should be adapted to the neuromelaninsensitivity level of the pulse sequence. To address this specific issue, Schwarz et al. [18] proposed a normalization procedure based on the theoretical volume of the SNpc hyperintense area in healthy controls. Because we wanted to test independently the accuracy of the U-net in the external dataset, we did not try to normalize the signal intensity level.

Another finding is that the accuracy of the SNpc segmentation was consistently lower than that of the midbrain, denoting the difficulty in determining the boundary of the SNpc regardless of the segmentation method. Unlike the boundaries between the midbrain and surrounding cisterns, the boundaries between the SNpc and the background are difficult to delineate precisely because hyperintense pixels depict only neuromelanin content and not the entire SNpc. The relative subjectivity inherent in the manual segmentation of the SNpc seems to have affected both manual and U-net segmentation accuracies. Further, the DSCs were lower in the patient group compared to the healthy group probably because reducedhyperintense areas result in an even more challenging segmentation task.

Despite the relative lack of precision of the SNpc segmentation in the external dataset, the calculated hyperintense areas were significantly reduced in patients with PD compared to $\mathrm{HC}$ in both datasets, consistent with the results of previous studies $[13,15,19,20]$. The diagnostic test accuracy for PD of the thresholding method was not affected: AUC were similar using U-net or manual segmentation in both datasets, with a slight comparative advantage for the U-net method, and as high to that of the previously described manual techniques, where it ranged from 0.82 to $0.93[13,15,20]$. These results suggest that an extremely precise segmentation of the $\mathrm{SNpc}$ is not required to provide useful size estimates of the hyperintense area. Our U-net model is sufficient to obtain a satisfying diagnostic accuracy.

The hyperintense areas were correlated to motor severity (reflected by UPDRS-III scores) in the external validation dataset but not in the larger principal dataset. We do not have a clear explanation for this finding, as disease severity was similar between the two groups. Due to its small size (12 PD patients), the correlation analyses performed on the external validation dataset should be viewed cautiously. As previous studies on smaller samples also found weak [18] or no correlation $[13,17]$ with UPDRS-III scores, the utility of NM-MRI as a monitoring tool for patients with PD could not be proved.

This study had several limitations. First, the sample size was relatively small for a case-control study, particularly of the external validation dataset. Second, PD diagnosis in this study was not histopathologically confirmed; thus, misdiagnosis could be possible. Third, as pathological examination could not be used as a criterion, the U-net model was trained using manually obtained masks of the SNpc and midbrain from NM-MRI as input. MS relies on recognition of the hyperintense area and the anatomical knowledge of the radiologist and is therefore subject to subjectivity bias. Hyperintense areas could be underestimated in patients with PD, amplifying the difference between the patients with PD and HC. Thus, additional sequences providing clearer SNpc images, such as proton density-weighted images, could be beneficial for creating more accurate SNpc masks for application to NM-MR images. Fourth, both the methods relied on a threshold to define the hyperintense area. A drawback of this approach is the loss of information, such as the magnitude of the signal intensity above the threshold or its spatial distribution [30]. Several studies have found sub-regional patterns of neuromelanin loss within the SNpc using manually placed regions of interest $[16,18]$ or voxel-wise analysis [30], with differences between $\mathrm{HC}$ and patients with PD preferentially involving the posterior and lateral parts of the SNpc. Studying the whole SNpc could have contributed to the lack of correlation with the clinical status in our study, which remains an important focus for further improvement of NM-MRI. Additional studies focusing on this region of the SNpc could help achieve this goal. Finally, mean disease duration was longer in the external validation dataset and it may have influenced positively the diagnostic accuracy of the method in the external dataset. Additionally, the mean age differed between the datasets; thus, the potential influence from these factors cannot be ignored because the midbrain is subject to agerelated changes [31]. However, despite these limitations, because the U-net saves times and does not affect the diagnostic accuracy of the thresholding method, it may be useful to promote the clinical application of NM-MRI for PD diagnosis.

In conclusion, U-net segmentation provided relatively high accuracy in the evaluation of the SNpc in NM-MRI and yielded diagnostic performance comparable to that of the established manual method, but its segmentation accuracy should be further improved to be able to fully replace manual segmentation.

Funding This study was funded by the Brain/MINDS Beyond program from the Japan Agency for Medical Research and Development (AMED) under Grant Number JP19dm0307024; JSPS KAKENHI (JP19K17244); a High Technology Research Center Grant from the Ministry of Education, Culture, Sports, Science, and Technology of Japan (MEXT); and the MEXT-Supported Program for the Strategic Research Foundation at Private Universities, 2014-2018.

\section{Compliance with ethical standards}

Conflict of interest The authors declare that they have no conflict of interest. 
Ethical approval All procedures performed in studies involving human participants were in accordance with the ethical standards of the institutional and/or national research committee and with the 1964 Helsinki declaration and its later amendments or comparable ethical standards.

Informed consent Informed consent was obtained from all individual participants included in the study.

Open Access This article is distributed under the terms of the Creative Commons Attribution 4.0 International License (http:// creativecommons.org/licenses/by/4.0/), which permits unrestricted use, distribution, and reproduction in any medium, provided you give appropriate credit to the original author(s) and the source, provide a link to the Creative Commons license, and indicate if changes were made.

\section{References}

1. Disease GBD, Injury I, Prevalence C (2018) Global, regional, and national incidence, prevalence, and years lived with disability for 354 diseases and injuries for 195 countries and territories, 19902017: a systematic analysis for the Global Burden of Disease Study 2017. Lancet 392(10159):1789-1858. https://doi.org/10.1016/ S0140-6736(18)32279-7

2. Drui G, Carnicella S, Carcenac C, Favier M, Bertrand A, Boulet S, Savasta M (2014) Loss of dopaminergic nigrostriatal neurons accounts for the motivational and affective deficits in Parkinson's disease. Mol Psychiatry 19(3):358-367. https://doi.org/10.1038/ mp. 2013.3

3. Tolosa E, Wenning G, Poewe W (2006) The diagnosis of Parkinson's disease. Lancet Neurol 5(1):75-86. https://doi.org/10. 1016/S1474-4422(05)70285-4

4. Cheng HC, Ulane CM, Burke RE (2010) Clinical progression in Parkinson disease and the neurobiology of axons. Ann Neurol 67(6):715-725. https://doi.org/10.1002/ana.21995

5. Lindholm D, Makela J, Di Liberto V, Mudo G, Belluardo N, Eriksson O, Saarma M (2016) Current disease modifying approaches to treat Parkinson's disease. Cell Mol Life Sci 73(7): 1365-1379. https://doi.org/10.1007/s00018-015-2101-1

6. Berardelli A, Wenning GK, Antonini A, Berg D, Bloem BR, Bonifati V, Brooks D, Burn DJ, Colosimo C, Fanciulli A, Ferreira J, Gasser T, Grandas F, Kanovsky P, Kostic V, Kulisevsky J, Oertel W, Poewe W, Reese JP, Relja M, Ruzicka E, Schrag A, Seppi K, Taba P, Vidailhet M (2013) EFNS/MDS-ES/ENS [corrected] recommendations for the diagnosis of Parkinson's disease. Eur J Neurol 20(1):16-34. https://doi.org/10.1111/ene.12022

7. Burciu RG, Ofori E, Archer DB, Wu SS, Pasternak O, McFarland NR, Okun MS, Vaillancourt DE (2017) Progression marker of Parkinson's disease: a 4-year multi-site imaging study. Brain 140(8):2183-2192. https://doi.org/10.1093/brain/awx146

8. Kamagata K, Hatano T, Okuzumi A, Motoi Y, Abe O, Shimoji K, Kamiya K, Suzuki M, Hori M, Kumamaru KK, Hattori N, Aoki S (2016) Neurite orientation dispersion and density imaging in the substantia nigra in idiopathic Parkinson disease. Eur Radiol 26(8): 2567-2577. https://doi.org/10.1007/s00330-015-4066-8

9. Sasaki M, Shibata E, Tohyama K, Takahashi J, Otsuka K, Tsuchiya K, Takahashi S, Ehara S, Terayama Y, Sakai A (2006) Neuromelanin magnetic resonance imaging of locus ceruleus and substantia nigra in Parkinson's disease. Neuroreport 17(11):12151218. https://doi.org/10.1097/01.wnr.0000227984.84927.a7

10. Trujillo P, Summers PE, Ferrari E, Zucca FA, Sturini M, Mainardi LT, Cerutti S, Smith AK, Smith SA, Zecca L, Costa A (2017)
Contrast mechanisms associated with neuromelanin-MRI. Magn Reson Med 78(5):1790-1800. https://doi.org/10.1002/mrm.26584

11. McGeer PL, Itagaki S, Boyes BE, McGeer EG (1988) Reactive microglia are positive for HLA-DR in the substantia nigra of Parkinson's and Alzheimer's disease brains. Neurology 38(8): 1285-1291. https://doi.org/10.1212/wnl.38.8.1285

12. Zhang W, Phillips K, Wielgus AR, Liu J, Albertini A, Zucca FA, Faust R, Qian SY, Miller DS, Chignell CF, Wilson B, JacksonLewis V, Przedborski S, Joset D, Loike J, Hong JS, Sulzer D, Zecca L (2011) Neuromelanin activates microglia and induces degeneration of dopaminergic neurons: implications for progression of Parkinson's disease. Neurotox Res 19(1):63-72. https://doi.org/ 10.1007/s12640-009-9140-Z

13. Castellanos G, Fernandez-Seara MA, Lorenzo-Betancor O, OrtegaCubero S, Puigvert M, Uranga J, Vidorreta M, Irigoyen J, Lorenzo E, Munoz-Barrutia A, Ortiz-de-Solorzano C, Pastor P, Pastor MA (2015) Automated neuromelanin imaging as a diagnostic biomarker for Parkinson's disease. Mov Disord 30(7):945-952. https://doi. org/10.1002/mds.26201

14. Kashihara K, Shinya T, Higaki F (2011) Neuromelanin magnetic resonance imaging of nigral volume loss in patients with Parkinson's disease. J Clin Neurosci 18(8):1093-1096. https://doi. org/10.1016/j.jocn.2010.08.043

15. Schwarz ST, Rittman T, Gontu V, Morgan PS, Bajaj N, Auer DP (2011) T1-weighted MRI shows stage-dependent substantia nigra signal loss in Parkinson's disease. Mov Disord 26(9):1633-1638. https://doi.org/10.1002/mds.23722

16. Ohtsuka C, Sasaki M, Konno K, Koide M, Kato K, Takahashi J, Takahashi S, Kudo K, Yamashita F, Terayama Y (2013) Changes in substantia nigra and locus coeruleus in patients with early-stage Parkinson's disease using neuromelanin-sensitive MR imaging. Neurosci Lett 541:93-98. https://doi.org/10. 1016/j.neulet.2013.02.012

17. Fabbri M, Reimao S, Carvalho M, Nunes RG, Abreu D, Guedes LC, Bouca R, Lobo PP, Godinho C, Coelho M, Goncalves NC, Rosa MM, Antonini A, Ferreira JJ (2017) Substantia nigra neuromelanin as an imaging biomarker of disease progression in Parkinson's disease. J Park Dis 7(3):491-501. https://doi.org/10. 3233/JPD-171135

18. Schwarz ST, Xing Y, Tomar P, Bajaj N, Auer DP (2017) In vivo assessment of brainstem depigmentation in Parkinson disease: potential as a severity marker for multicenter studies. Radiology 283(3):789-798. https://doi.org/10.1148/radiol.2016160662

19. Hatano T, Okuzumi A, Kamagata K, Daida K, Taniguchi D, Hori M, Yoshino H, Aoki S, Hattori N (2017) Neuromelanin MRI is useful for monitoring motor complications in Parkinson's and PARK2 disease. J Neural Transm (Vienna) 124(4):407-415. https://doi.org/10.1007/s00702-017-1688-9

20. Ogisu K, Kudo K, Sasaki M, Sakushima K, Yabe I, Sasaki H, Terae S, Nakanishi M, Shirato H (2013) 3D neuromelanin-sensitive magnetic resonance imaging with semi-automated volume measurement of the substantia nigra pars compacta for diagnosis of Parkinson's disease. Neuroradiology 55(6):719-724. https://doi. org/10.1007/s00234-013-1171-8

21. Aljabar P, Heckemann RA, Hammers A, Hajnal JV, Rueckert D (2009) Multi-atlas based segmentation of brain images: atlas selection and its effect on accuracy. Neuroimage 46(3):726-738. https:// doi.org/10.1016/j.neuroimage.2009.02.018

22. LeCun Y, Bengio Y, Hinton G (2015) Deep learning. Nature 521(7553):436-444. https://doi.org/10.1038/nature14539

23. Naceur MB, Saouli R, Akil M, Kachouri R (2018) Fully automatic brain tumor segmentation using end-to-end incremental deep neural networks in MRI images. Comput Methods Prog Biomed 166:39 49. https://doi.org/10.1016/j.cmpb.2018.09.007

24. Li H, Jiang G, Zhang J, Wang R, Wang Z, Zheng WS, Menze B (2018) Fully convolutional network ensembles for white matter 
hyperintensities segmentation in MR images. Neuroimage 183: 650-665. https://doi.org/10.1016/j.neuroimage.2018.07.005

25. Zhu W, Huang Y, Zeng L, Chen X, Liu Y, Qian Z, Du N, Fan W, Xie X (2019) AnatomyNet: deep learning for fast and fully automated whole-volume segmentation of head and neck anatomy. Med Phys 46(2):576-589. https://doi.org/10.1002/mp.13300

26. Ronneberger O, Fischer P, Brox T (2015) U-net: convolutional networks for biomedical image segmentation. arXiv:150504597

27. Postuma RB, Berg D, Stern M, Poewe W, Olanow CW, Oertel W, Obeso J, Marek K, Litvan I, Lang AE, Halliday G, Goetz CG, Gasser T, Dubois B, Chan P, Bloem BR, Adler CH, Deuschl G (2015) MDS clinical diagnostic criteria for Parkinson's disease. Mov Disord 30(12):1591-1601. https:// doi.org/10.1002/mds.26424

28. Kingma DP, Ba LJ (2014) Adam: a method for stochastic optimization. arXiv: 14126980

29. DeLong ER, DeLong DM, Clarke-Pearson DL (1988) Comparing the areas under two or more correlated receiver operating characteristic curves: a nonparametric approach. Biometrics 44(3):837-845

30. Sulzer D, Cassidy C, Horga G, Kang UJ, Fahn S, Casella L, Pezzoli G, Langley J, Hu XP, Zucca FA, Isaias IU, Zecca L (2018) Neuromelanin detection by magnetic resonance imaging (MRI) and its promise as a biomarker for Parkinson's disease. NPJ Park Dis 4:11. https://doi.org/10.1038/s41531-018-0047-3

31. Xing Y, Sapuan A, Dineen RA, Auer DP (2018) Life span pigmentation changes of the substantia nigra detected by neuromelaninsensitive MRI. Mov Disord 33(11):1792-1799. https://doi.org/10. $1002 /$ mds. 27502

Publisher's note Springer Nature remains neutral with regard to jurisdictional claims in published maps and institutional affiliations. 